\title{
DATA-DRIVEN MRSI SPECTRAL LOCALIZATION USING NON-CARTESIAN SAMPLING TRAJECTORIES
}

\author{
Jeffrey Kasten ${ }^{\star \dagger} \quad$ François Lazeyras ${ }^{\star} \quad$ Dimitri Van De Ville ${ }^{\star \dagger}$ \\ * Department of Radiology and Medical Informatics, University of Geneva, Switzerland \\ $\dagger$ Institute of Bioengineering, Ecole Polytechnique Fédérale de Lausanne, Switzerland
}

\begin{abstract}
The ability to non-invasively visualize spatially-localized maps of metabolite concentrations in vivo as afforded by Magnetic Resonance Spectroscopic Imaging (MRSI) is an attractive prospect in clinically-focused biomedical imaging. However, the current gold standard implementation, known as Chemical Shift Imaging (CSI), is plagued by various artifacts, due primarily to the limitations dictated through use of the Fourier transform. To counter these impediments, numerous "constrained" reconstruction methods have been suggested, which typically inject some type of a priori information, usually with the aid of structural MR images, into the signal model. While this may be desirable for some applications, it introduces an assumption which posits a general equivalency between the spatial and spectral distributions, which may not always be appropriate. This work examines an alternative formulation in which, with the aid of statistical techniques and spatial regularization, constituent highresolution spatial and spectral components are estimated from the raw MRSI data. We demonstrate the efficacy of this technique, and the robustness of the estimated components to alternative sampling strategies, thereby broadening the applicability of the method and offering the prospect of reduced acquisition times in more pressed clinical settings.
\end{abstract}

Index Terms - Magnetic Resonance Spectroscopic Imaging, Chemical Shift imaging, Principal Component Analysis, Total Variation, Constrained Reconstruction

\section{INTRODUCTION}

In a MRSI experiment, the measured signal at $k$-space location $\mathbf{k}_{i}, i=1, \ldots, M$ and time $t$ can be expressed as:

$$
s\left(\mathbf{k}_{i}, t\right)=\int_{-\infty}^{\infty} \int_{F O V} \rho(\mathbf{x}, f) e^{-2 \pi j\left(\mathbf{k}_{i} \cdot \mathbf{x}+f t\right)} d \mathbf{x} d f
$$

where

$$
\mathbf{k}_{i}=\mathbf{k}\left(t_{i}^{\prime}\right)=\frac{\gamma}{2 \pi} \int_{0}^{t_{i}^{\prime}} \mathbf{G}(\tau) d \tau
$$

represents the employed sampling trajectory determined by the gradients, $\mathbf{G}$, and $\rho(\mathbf{x}, f)$ the spatio-spectral distribution of the imaged object. For completeness, $\mathbf{x}$ denotes position in the spatial domain, $f$ the temporal frequency, $\gamma$ the gyromagnetic ratio, and FOV the fieldof-view. In a standard CSI setting [1] in 2D, a free induction decay (FID) is acquired at each $\mathbf{k}_{i}$, such that the total measurement time

This work was supported in part by the Swiss National Science Foundation (under grants 320030-135425 and PP00P2-123438) and in part by the Center for Biomedical Imaging (CIBM). is $T_{\text {acq }}=N_{x} N_{y} T_{R}$, where $N_{x}$ and $N_{y}$ represent the total number of encodings in each Cartesian dimension, and $T_{R}$ is the repetition time. In most clinical settings, the number of encodings are kept low because of practical time considerations, and typically only values of $\mathbf{k}_{i}$ corresponding to low spatial frequencies are measured. For traditional Fourier-based reconstructions, this effectively corresponds to an assumption that the imaged object is band-limited to the extent of the acquired measurements, which introduces spectral contamination artifacts due to a broadened pointspread function. In order to reduce these artifacts, numerous alternatives have been proposed which seek to improve the problem conditioning, using such methods as sensitivity encoding [2], alternative sampling strategies [3,4], or modeling the spatio-spectral distribution function with the aid of additional MR modalities [5-7]. Given that the majority of MR scanning sessions routinely include some type of high-resolution structural scans, the latter approach may seem particularly attractive, as it offers the ability to incorporate high-resolution a priori information into the reconstruction. However, such an approach can introduce additional artifacts into the reconstruction when the spatial and spectral data contain contrasting information. To circumvent this potential pitfall, we propose a variation whereby the signal model is estimated directly from the MRSI data, relying upon a few general assumptions and necessitating only a map of the static $B_{0}$ field as a supplemental modality. Moreover, we show that our method is robust to non-Cartesian sampling strategies, which further paves the way for faster and more efficient acquisitions.

\section{COMPONENT-BASED MRSI RECONSTRUCTION}

\subsection{Spatio-spectral model}

In methods such as SLIM [5], the spatio-spectral distribution function is modeled as a sum of $K$ spectrally-homogeneous compartments, $\chi_{k}(\mathbf{x})$ (typically derived from segmented structural images), times their associated spectrum, $q_{k}(f)$ :

$$
\rho_{\text {SLIM }}(\mathbf{x}, f)=\sum_{k=1}^{K} q_{k}(f) \chi_{k}(\mathbf{x}),
$$

or when the static inhomogeneity profile, $\Delta f(\mathbf{x})=\frac{\gamma}{2 \pi} \Delta B_{0}(\mathbf{x})$, is known, as the BSLIM model [7]:

$$
\rho_{\text {BSLIM }}(\mathbf{x}, f)=\sum_{k=1}^{K} q_{k}(f-\Delta f(\mathbf{x})) \chi_{k}(\mathbf{x}) .
$$


Electing to work with the former expression, the imaging equation (1) can then be reduced to a linear system of equations:

$$
\begin{aligned}
& \sum_{k=1}^{s_{\text {SLIM }}\left(\mathbf{k}_{i}, t\right)=} \underbrace{\int_{-\infty}^{\infty} q_{k}(f) e^{-2 \pi j f t} d f}_{Q_{k}(t)} \cdot \underbrace{\int_{\text {FOV }} \chi_{k}(\mathbf{x}) e^{-2 \pi j\left(\mathbf{k}_{i} \cdot \mathbf{x}\right)} d \mathbf{x}}_{K_{k}\left(\mathbf{k}_{i}\right)} \\
& =\sum_{k=1}^{K} Q_{k}(t) K_{k}\left(\mathbf{k}_{i}\right),
\end{aligned}
$$

where $Q_{k}(t)$ is the FID originating from the $k^{\text {th }}$ compartment, and $K_{k}\left(\mathbf{k}_{i}\right)$ are the SLIM kernels. One immediate advantage of this type of model is that it obviates the rectangular voxel restriction stipulated by the Fourier transform by permitting the use of the compartments as "generalized voxels," which can be arbitrarily defined.

Assuming the validity of (3), (4) as appropriate signal models, it is natural to ask whether a decomposition such as (6) could be obtained without imposing an explicit basis set, $\chi_{k}(\mathbf{x})$. An intuitive choice of venue would include Principal Component Analysis [8] (implemented as a singular value decomposition (SVD)), which produces a bilinear decomposition of the measured data in $\mathbf{k}$ and $t$, such that the variance of the resulting components is maximized, subjected to an orthogonality constraint, i.e.,

$$
s_{\mathrm{SVD}}\left(\mathbf{k}_{i}, t\right)=\sum_{k=1}^{K} \sigma_{k} u_{k}\left(\mathbf{k}_{i}\right) v_{k}(t), \quad i=1, \ldots, M .
$$

Here, $v_{k}(t)$ can be regarded as the "eigen-FIDs," and $u_{k}\left(\mathbf{k}_{i}\right)$ as their corresponding spatial distribution ("eigen-maps").

\subsection{Variational Framework for Determining High-Resolution Components}

Ideally, we would like to attain spatial maps $\tilde{u}_{k}(\mathbf{x})$ at a higher resolution (defined in our case to be that of the acquired inhomogeneity profile, discussed further in section 2.3), given only the lowresolution $k$-space components, $u_{k}\left(\mathbf{k}_{i}\right)$. This problem is ill-posed, and so additional regularization is required. Use of the total variation (TV) semi-norm has become prevalent in various MR reconstruction problems, primarily due to its proclivity for retaining sharp edges, and is quite appropriate for our application. In particular, we adopt the method of [10], utilizing the total generalized variation $\left(\mathrm{TGV}^{2}\right)$, which favors piecewise linear solutions by compromising between sparsity in the first and second derivatives. Formally, it is expressed as:

$$
\operatorname{TGV}_{\alpha}^{2}\{\zeta\}=\underset{h}{\operatorname{minimize}} \alpha_{1} \int_{\Omega}|\nabla \zeta(\mathbf{x})-h| d \mathbf{x}+\alpha_{0} \int_{\Omega}|\mathcal{E}\{h\}| d \mathbf{x},
$$

where $\mathcal{E}\{h\}=\frac{1}{2}\left(\nabla h+\nabla h^{\mathrm{T}}\right)$. We therefore formulate the recovery of high-resolution components as the convex optimization problem:

$$
\begin{aligned}
& \underset{\tilde{u}_{k}}{\operatorname{minimize}} \mathcal{J}\left\{\tilde{u}_{k}\right\}=\left\|\mathcal{A} \tilde{u}_{k}-u_{k}\right\|_{\ell_{2}}^{2}+\lambda \mathrm{TGV}_{\alpha}^{2}\left\{\tilde{u}_{k}\right\}, \\
& k=1, \ldots, K
\end{aligned}
$$

where $\mathcal{A}$ is the so-called "system matrix," (sampling operator) which depends on the particular sampling strategy. For our experiments, values of $\alpha_{1}=1.0 \times 10^{-3}$ and $\alpha_{0}=2 \alpha_{1}$ were determined empirically.

\subsection{Static Field Inhomogeneity Compensation}

It is important to note that the components generated in (7) will include any effects precipitated by imperfect measurement conditions, primarily those due to local $B_{0}$ distortions. To compensate, an additional measurement of the static field inhomogeneity, $\Delta f(\mathbf{x})$, at high spatial resolution is necessary, e.g., using the method of [9]. Inspired by BSLIM, we can then define an operator, $\mathscr{D}_{\Delta f}$, which spectrally redistributes the spatial maps along the temporal frequency axis (chemical shift dimension). The formal definition is given below:

Definition 1 Given a spatial inhomogeneity profile, $\Delta f(\boldsymbol{x})$, the spectral deformation operator, $\mathscr{D}_{\Delta f}$, acting upon an image, $\varphi$, is given by:

$$
\mathscr{D}_{\Delta f}\{\varphi\}:=h(\boldsymbol{x}, f)=\varphi(\boldsymbol{x}) \delta(f-\Delta f(\boldsymbol{x})) .
$$

As we ultimately work with discrete data, we denote the discretized version of this operator as:

$$
h\left[\mathbf{x}_{n}, f_{l}\right]=\left(\mathscr{D}_{\Delta f}\{\varphi\}(\mathbf{x}, f) * \beta^{1}\left(f \frac{T}{F_{s}}\right)\right)\left[\mathbf{x}_{n}, f_{l}\right],
$$

where $F_{s}$ is the temporal sampling rate of the acquisition scheme, $T$ the number of samples collected for each FID, and $\beta^{1}$ a first degree B-spline (linear interpolation). We subsequently denote discrete measurements through the use of square brackets.

\subsection{Solving for Compensated Components}

With all the elements now in place, we can solve for the static field inhomogeneity compensated components in the following way. We first unwind the effect of $\Delta f\left[\mathbf{x}_{n}\right]$ by applying the spectral deformation operator with a flip of sign: $\mathscr{D}_{-\Delta f}$ on the initial, high-resolution spatial maps: $\hat{h}_{k}\left[\mathbf{x}_{n}, f_{l}\right]=\mathscr{D}_{-\Delta f}\left\{\tilde{u}_{k}\right\}$. Next, we generate the full kernel matrix,

$$
\hat{H}_{k}\left[\mathbf{k}_{i}, t_{m}\right]=\mathcal{F}_{t}^{-1}\left\{\mathcal{A}\left\{\hat{h}_{k}\right\}\right\}, \quad k=1, \ldots, K,
$$

where $\mathcal{F}_{t}$ denotes the temporal Fourier transform. We then re-form the measurement matrix, such that:

$$
\begin{aligned}
& s^{\prime}\left[\mathbf{k}_{i}, t_{m}\right]=\sum_{k=1}^{K} \sigma_{k} \hat{H}_{k}\left[\mathbf{k}_{i}, t_{m}\right] v_{k}\left[t_{m}\right], \\
& i=1, \ldots, M, \quad m=1, \ldots, T .
\end{aligned}
$$

This new measurement matrix should be devoid of any static field inhomogeneity effects, and a subsequent SVD should yield undistorted components, $u_{k}^{\prime}$ and $v_{k}^{\prime}$, in this respect. Accordingly, a final determination of the high-resolution spatial components, $\tilde{u}_{k}^{\prime}$ as in (9) will yield solutions which reflect our two general assumptions: (a) that the model given by (3) is indeed an accurate description of the imaged object, and $(b)$ that the $\chi_{k}(\mathbf{x})$ (or in our decompositions, $\tilde{u}_{k}$, $\tilde{u}_{k}^{\prime}$ ) are piecewise linear functions that may exhibit sharp boundaries.

\section{SIMULATED EXPERIMENTS}

In this section, we demonstrate reconstructions using an implementation of the above routine on simulated MRSI data. We show that our method can recover inhomogeneity-compensated components corresponding to the "true" underlying distributions, and that these 

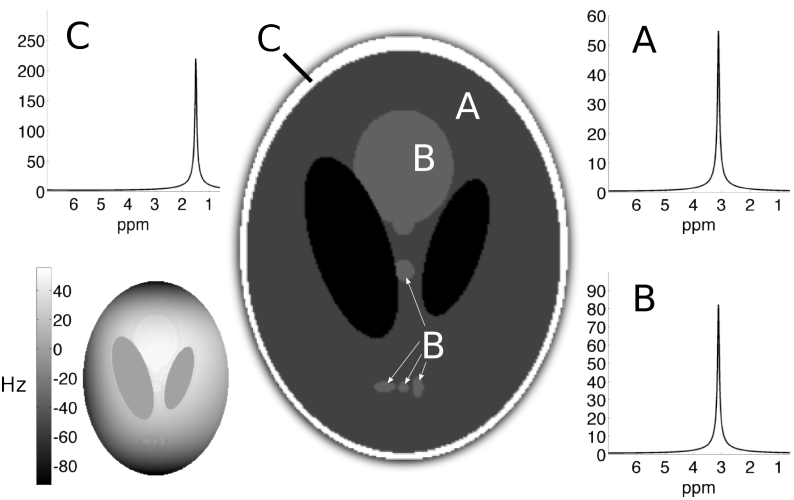

Fig. 1. Simulated phantom composition and associated static $B_{0}$ profile. All spectra are shown in magnitude.

components are determined in a robust manner that remains independent of acquisition type.

The simulated phantom geometry is shown in Figure 1 (a modified Shepp-Logan phantom). To each compartment, a unique spectrum was associated with fixed temporal resolution ( $T=1024$ sample points). A $B_{0}$ static inhomogeneity profile, chosen to mimic those typically observed in ${ }^{1} \mathrm{H}$ MRSI experiments using geometrically similar phantoms, was also simulated using a fourth-degree polynomial model, discretized on the desired spatial grid of the reconstructed MRSI data (in our case $256 \times 256$ ). Simulations were conducted using two different sampling strategies: standard Cartesian and spiral. For the Cartesian sampling scheme, $M=1024$ sample points, corresponding to a $32 \times 32$ sampling grid was chosen, with a FOV of $([-0.14 \ldots 0.14] \times[-0.14 \ldots 0.14])$ meters. For the spiral case, three interleaved constant angular velocity spiral trajectories were acquired, using the same FOV as in the Cartesian case, such that the total number of measurements remained equal to $M$. As a final step, white Gaussian noise was added to the simulated $k$-space measurements, yielding a mean SNR of $21.83 \mathrm{~dB}$.

For each sampling scheme, the forward and adjoint sampling operators, $\mathcal{A}$, and $\mathcal{A}^{*}$, respectively, were implemented using the NUFFT (non-uniform fast Fourier transform), as described in [11], thereby seeking a compromise between computational speed and numerical accuracy. Furthermore, for the spiral acquisition scheme, an additional density compensation function (DCF) is needed to account for non-uniform sampling. In our experiments, we took this to be the area of the Voronoi cell around each sampling point, as proposed in [12]. The DCF also enters into the component estimation by the generalized singular value decomposition (GSVD), where $\mathbf{X}=\overline{\mathbf{U}} \boldsymbol{\Sigma} \mathbf{V}^{H}$ such that $\overline{\mathbf{U}}^{H} \mathbf{W} \overline{\mathbf{U}}=\mathbf{I}$. In our case, $\mathbf{W}$ is a diagonal matrix containing the DCF weightings for each $k$-space position. Note that in uniform Cartesian sampling, this reduces to the normal SVD by setting $\mathbf{W}=\mathbf{I}$. Upon initialization of the reconstruction scheme, the number of components (i.e. the working subspace), must be chosen. An appropriate choice of dimensionality is a general ongoing discussion, and in this work we use the method of [13], which maximizes the Bayesian evidence in a probabilistic PCA model. In each dataset, the initial underlying (noninhomogeneity-compensated) dimensionality was determined to be $K=40$.

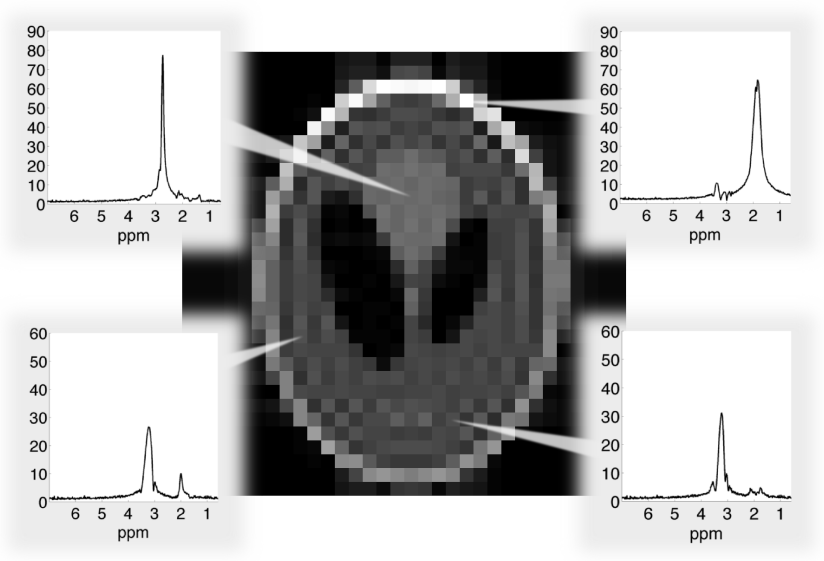

Fig. 2. Standard inverse Fourier reconstruction. A few representative spectra are shown (magnitude), overlaid on a magnitude image created by plotting the first sample point of each FID.

\begin{tabular}{c|c|c|c|c|c|c|}
\hline \multirow{2}{*}{} & \multicolumn{2}{|c|}{ Cartesian } & \multicolumn{2}{c|}{ Spiral } & \multicolumn{2}{c|}{$\begin{array}{c}\text { Spiral } \\
\text { (20\% sub-sampling) }\end{array}$} \\
\cline { 2 - 7 } & ROI1 & ROI2 & ROI1 & ROI2 & ROI1 & ROI2 \\
\cline { 2 - 7 } Spatial Map & 22.64 & 19.88 & 23.16 & 20.65 & 22.76 & 20.14 \\
Spectrum & 26.71 & 25.76 & 27.83 & 27.18 & 27.12 & 26.29 \\
\hline
\end{tabular}

Table 1. PSNR values for composite spatial maps and spectra $(\mathrm{dB})$

\section{RESULTS AND VALIDATION}

Because the SVD operates under an orthogonality constraint, the spatial and spectral components generated by the reconstruction may in fact remain linear combinations of the true spectrallyhomogeneous compartments. Hence, further treatment will likely be application-specific. For example, an operator may view the resultant components, and wish to examine the spectrum within a particular region of interest (ROI). Once defined, the ROI may be used for linear regression in order to determine the required spatial component weightings, which may in turn be applied to the corresponding spectral components to achieve the best within-ROI spectrum.

In order to validate the method, and having access to the true spatio-spectral distribution, we defined as ROI1 regions A+B in Figure 1 and ROI 2 as region $\mathrm{C}$. For the linear regression, we selected the first five estimated spatial components, $\left\{\tilde{u}_{1}^{\prime}, \tilde{u}_{2}^{\prime}, \ldots, \tilde{u}_{5}^{\prime}\right\}$, for each sampling scheme, corresponding to the largest singular values. Composite high-resolution spatial maps and spectra were then created from the components using the regression weightings, and were subsequently scaled to have unit $\ell_{2}$ norm. As an initial reference, results generated by standard inverse Fourier reconstruction are provided in Figure 2. The results for each sampling strategy using the proposed method are displayed in Figure 3 along with the associated $k$-space trajectories. As further validation, the defined ROIs were also scaled to unit $\ell_{2}$ norm, and the PSNR for each composite spatial map and spectrum was computed. The same analysis was also completed for a sub-sampled version of the spiral trajectory, such that the outer $20 \%$ of $k$-space measurements were discarded. The results are collected in Table 1. 

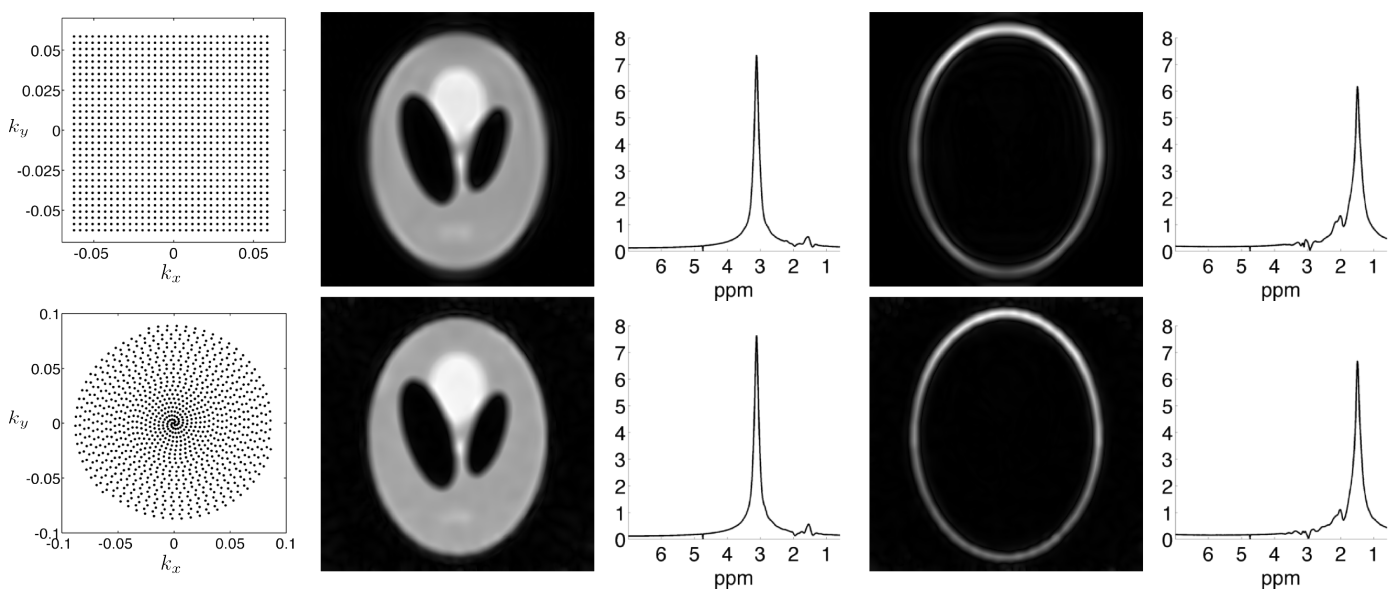

Fig. 3. Reconstruction results for Cartesian (top) and spiral (bottom) trajectories. All images and spectra are displayed in magnitude.

\section{DISCUSSION AND CONCLUSION}

The results in Figure 3 clearly show that the proposed method is able to recover the underlying spatio-spectral distribution of the imaged object. Moreover, the composite spectra are located at their correct resonance positions, though some lineshape distortion can be seen, especially in ROI2. This result can likely be explained by both the fact that the inhomogeneity profile is particularly pronounced within this ROI (at the phantom periphery), and by observing that a large proportion of the energy in the Fourier domain lies well beyond the sampled region. A small contamination in the ROI1 spectrum from ROI2 can also be seen, though minimal given the amplitude discrepancy between the ground truth spectra in the two regions.

Both a visual comparison in Figure 3 and the results in Table 1 indicate superior reconstruction quality is achieved when using a spiral trajectory. This is not an unexpected outcome, as spiral trajectories offer a number of advantages over traditional Cartesian sampling schemes [14], such as more efficient $k$-space coverage by not spending time covering the corners. Thus, given a measurement budget of $M$ points, the spiral sampling scheme is able to probe more distal regions in $k$-space and improve the reconstruction problem conditioning. A further benefit of the proposed reconstruction method is that it remains compatible with other accelerated imaging techniques such as SENSE and GRAPPA [15], offering the possibility of yet improved reconstructions through the use of multiple receiver coils.

In this work we have presented a reconstruction method for MRSI data that circumvents a number of shortcomings associated with traditional inverse Fourier reconstructions, as well as the ambiguity in selecting appropriate a priori compartmental information in SLIM-like approaches. The geometric assumptions made on the recovered spatial distributions are general enough to accommodate most biological samples of interest, and the approach is flexible enough to be used in conjunction with a wide array of acquisition and measurement acceleration techniques.

\section{REFERENCES}

[1] T. R. Brown, B. M. Kincaid, and K. Ugurbil, "NMR chemical shift imaging in three dimensions.", Proceedings of the $\mathrm{Na}$ tional Academy of Sciences of the United States of America, vol. 79, no. 11, pp. 3523-3526, 1982.

[2] K. P. Pruessmann, M. Weiger, M. B. Scheidegger, and P. Boesiger, "SENSE: sensitivity encoding for fast MRI,", Magn Reson Med, vol. 42, pp. 952-962, Nov 1999.
[3] S. Uribe, A. Guesalaga, R. Mir, M. Guarini, and P. Irarrzaval, "A 3D trajectory for undersampling k-space in MRSI applications," Magnetic Resonance Imaging, vol. 25, no. 3, pp. 350 358, 2007.

[4] D.-H. Kim, R. Henry, and D. M. Spielman, "Fast multivoxel two-dimensional spectroscopic imaging at 3 T," Magnetic Resonance Imaging, vol. 25, no. 8, pp. 1155 - 1161, 2007.

[5] X. Hu, D. N. Levin, P. C. Lauterbur, and T. Spraggins, "SLIM: spectral localization by imaging.," Magn Reson Med, vol. 8, pp. 314-322, Nov 1988.

[6] Z. P. Liang and P. C. Lauterbur, "A generalized series approach to MR spectroscopic imaging.," IEEE Trans Med Imaging, vol. 10, no. 2, pp. 132-137, 1991.

[7] I. Khalidov, D. Van De Ville, M. Jacob, F. Lazeyras, and M. Unser, "BSLIM: Spectral localization by imaging with explicit $B_{0}$ field inhomogeneity compensation," IEEE Transactions on Medical Imaging, vol. 26, no. 7, pp. 990-1000, 2007.

[8] K. Pearson, "On lines and planes of closest fit to systems of points in space," Philosophical Magazine, vol. 2, no. 11, pp. 559-572, 1901.

[9] P. Jezzard and R. S. Balaban, "Correction for geometric distortion in echo planar images from B0 field variations," Magn Reson Med, vol. 34, pp. 65-73, Jul 1995.

[10] F. Knoll, K. Bredies, T. Pock, and R. Stollberger, "Second order total generalized variation (TGV) for MRI," Magnetic Resonance in Medicine, vol. 65, no. 2, pp. 480-491, 2011.

[11] J. Fessler and B. Sutton, "Nonuniform Fast Fourier Transforms Using Min-Max Interpolation," Signal Processing, IEEE Transactions on, vol. 51, pp. 560 - 574, feb 2003.

[12] V. Rasche, R. Proksa, R. Sinkus, P. Börnert, and H. Eggers, "Resampling of Data Between Arbitrary Grids Using Convolution Interpolation," Medical Imaging, IEEE Transactions on, vol. 18, pp. 38-392, may 1999.

[13] T. P. Minka, "Automatic choice of dimensionality for PCA," Advances in neural information processing systems, vol. 13, no. 514, pp. 598-604, 2001.

[14] B. M. Delattre, R. M. Heidemann, L. A. Crowe, J.-P. Vallée, and J.-N. Hyacinthe, "Spiral demystified," Magnetic Resonance Imaging, vol. 28, no. 6, pp. 862 - 881, 2010.

[15] M. A. Griswold, P. M. Jakob, R. M. Heidemann, M. Nittka, V. Jellus, J. Wang, B. Kiefer, and A. Haase, "Generalized autocalibrating partially parallel acquisitions (GRAPPA)," Magn Reson Med, vol. 47, pp. 1202-1210, Jun 2002. 